Original Article

\title{
Is poor proprioception associated with worse movement quality of the knee in individuals with anterior cruciate ligament deficiency or reconstruction?
}

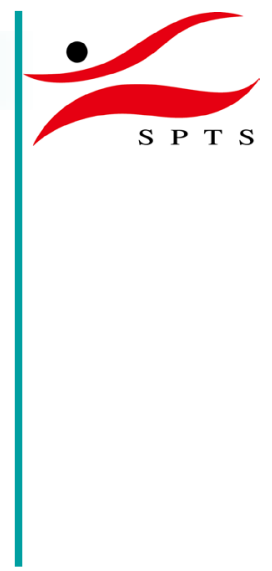

\author{
Anna Cronström, PT, $\mathrm{PhD}^{1)}$ \\ 1) Department of Health Sciences, Lund University: PO Box 157, SE-221 00 Lund, Sweden
}

\begin{abstract}
Purpose] To investigate the association between proprioception and a knee medial to foot position during weight-bearing activities in patients with anterior cruciate ligament reconstruction and anterior cruciate ligament deficiency. [Participants and Methods] Thirty-eight patients with anterior cruciate ligament reconstruction and 13 patients with anterior cruciate ligament deficiency aged 18-40 years were included. Proprioception was assessed by knee kinesthesia measured by the threshold to detection of passive motion. Movement quality was assessed by visual observation of the position of the knee in relation to the foot during the mini squat, the single-leg hop for distance, and the cross-over hop for distance. [Results] In patients with anterior cruciate ligament deficiency, worse kinesthesia was significantly associated with a knee medial to foot position during the single-leg hop for distance. No statistically significant associations were observed between kinesthesia and a knee medial to foot position during the three tasks in patients with anterior cruciate ligament reconstruction. [Conclusion] Poor proprioception may be associated with worse movement quality of the knee in patients with anterior cruciate ligament deficiency, but not in patients with anterior cruciate ligament reconstruction. Differences in sensorimotor function between patients with reconstructed and non-reconstructed anterior cruciate ligament should be considered in training and rehabilitation regimens aiming to enhance performance and prevent further injuries in these patients.

Key words: Anterior cruciate ligament injury, Proprioception, Movement quality
\end{abstract}

(This article was submitted May 28, 2018, and was accepted Jul. 26, 2018)

\section{INTRODUCTION}

Increased knee abduction or a knee medial to foot position (KMFP) during weight-bearing activity is commonly reported after anterior cruciate ligament deficiency (ACLD) or reconstruction (ACLR) ${ }^{1-4)}$ and might still be observed 20 years after injury ${ }^{4}$. This altered movement pattern is consistently reported as a part of the mechanism of non-contact ACL injury ${ }^{5-7)}$ and was found associated with greater strain on the $\mathrm{ACL}^{8-10)}$. Furthermore, increased knee abduction was previously suggested to be associated with worse hop performance ${ }^{11)}$ and an increased risk of subsequent ACL injuries to either knee ${ }^{12,13)}$. However, most studies investigating the underlying factors of this movement pattern have been focused on healthy individuals; hence, this issue is poorly studied in patients with ACL injury ${ }^{14)}$.

Several factors, such as gluteus maximus activation, trunk strength, and ankle range of motion, have been reportedly associated with knee abduction in healthy individuals ${ }^{14}$. However, given the reported alterations in sensorimotor function after ACL injury ${ }^{15-18)}$, it is possible that the factors contributing to knee abduction in this group of patients could be different from those observed in healthy individuals. For example, some well-known deficits in proprioceptive acuity as measured by the knee kinesthesia or joint position sense after the ACL injury ${ }^{17)}$ may persist even after returning to high-level sports ${ }^{19)}$. The 
proprioceptive receptors situated in the ACL are important for joint stability ${ }^{20)}$ and may play a role in controlling the sagittal and frontal plane knee movements ${ }^{21}{ }^{22}$. In a previous study ${ }^{22}$, we found that worse knee kinesthesia was associated with a KMFP during drop jump in participants with ACLD or ACLR. However, the participants with ACLD and ACLR included in that study were pooled in the analysis. Several differences in sensorimotor function between patients with ACLD and ACLR were reported. For example, patients with ACLD exhibit poorer proprioception ${ }^{17)}$ and use different postural and motor control strategies compared to those with $\mathrm{ACLR}^{23}$, 24). Poor kinesthesia has also been found related to worse self-reported function in patients with ACLD but not after ACLR ${ }^{25}$. Thus, separate analysis of patients with ACLD and ACLR may be warranted in studies on sensorimotor function after ACL injury. This exploratory study constitutes a secondary analysis of a previous study ${ }^{22}$. The aim of this study was to investigate the association between knee kinesthesia and visually assessed medio-lateral knee position during three functional tasks in patients with ACLD and ACLR.

\section{PARTICIPANTS AND METHODS}

As a sample of convenience, 13 individuals with ACLD and 38 individuals that had undergone ACLR, all from a previous study ${ }^{22}$, were included in this report. Included patients were aged 18-40 years, with or without associated injuries of the knee, and undergoing current rehabilitation training at a physiotherapy clinic. Patients that used crutches or had other injuries affecting the lower limb function more than the knee injury were excluded. Participants' characteristics are presented in Table 1. All patients were recruited at the same sports injury clinic (Kulan idrottsskadecentrum) in Skåne, Sweden and were undergoing the same rehabilitation program for ACL injury. All patients provided written informed consent prior to inclusion. The study was approved by the Advisory Committee for Research Ethics in Health Education at the Faculty of Medicine, Lund University (VEN 48-12).

Three tasks with different degrees of difficulty (mini-squat, single-leg hop for distance [SLHD], and cross-over hop for distance [COHD]) were used in the analysis. A 5-minute warm-up on a stationary bike preceded the evaluation of the functional tasks. All participants wore shorts and performed the mini-squat barefoot and the SLHD and COHD wearing their personal trainers. The knee kinesthesia was assessed by an investigator who was blinded to the movement quality scores. A consensus assessment, reached by two raters who were blinded to the kinesthesia measurements was used for evaluating medio-lateral knee position scores. Three practice trials were allowed for each task and data for the injured leg was used in the analysis.

Kinesthesia was measured by the Threshold to Detection of Passive Motion (TDPM) on a specially designed platform, as previously described ${ }^{26)}$. The platform was mounted on a steel frame and had an electric motor with a wire mounted at the end. The participant laid on the platform in a lateral position with the injured leg in a plastic splint. The splint was attached to a sled and connected to the wire that could make the splint move the knee in either flexion or extension at an angular speed of 1 degree per second. An analog scale at the end of the platform was used to register movements in increments of 0.25 degrees. In order to prevent any false movements induced by the sound produced by the start of the measuring device, all participants wore headphones and listened to a recording of the sound that was produced by the electric motor. The participants were told to close their eyes and indicate by raising their hand when they felt any movement of the knee. TDPM was measured towards extension (TE) and flexion (TF) from a 20-degree starting position ${ }^{26}$. The median value of three consecutive measurements of TE2 0 and TF20 was determined. An index value created from the sum of TE20 and TF20 was used for statistical analysis. A higher value indicated poorer TDPM. Moderate reliability ( $\mathrm{ICC}=0.63-0.70)$ has been previously reported for this device ${ }^{26)}$.

The mini squat was performed as described by Trulsson et al. ${ }^{2}$, modified to not exceed 90 degrees of the knee flexion. Participants were instructed to stand on a marked line with the feet hip-width apart and arms free. They were then asked to flex the knees to approximately 80 degrees and then rise up again. The consensus assessment from three trials was used in the analysis.

The participants performed the single-leg hop for distance with arms free ${ }^{27)}$. The participants were told to stand on the injured leg with the toes behind a marked line and with the other leg lifted from the floor. They were then instructed to jump as far as possible, taking off and landing on the same foot (arm swing allowed), and maintaining balance for 2-3 seconds. Three trials were performed. If the third jump was the longest one, additional jumps were performed until no increase was seen. The consensus assessment from the three trials was used in the analysis.

The COHD was performed as previously described ${ }^{28,29)}$. The participants stood on the injured leg with the other leg lifted from the floor. The supporting leg was placed with the toes at the start of a 6-meter marked line. The participants were then instructed to jump forward as far as possible diagonally crossing the line three consecutive times, taking off and landing on the same leg in each jump, and maintain their balance for 2-3 seconds during the last landing. The consensus assessment from three jumps was used in the analysis.

Medio-lateral knee position was assessed by visual observation of a video recording of each trial. The position of the knee in relation to the foot was independently scored by two experienced physiotherapists during each functional task, on a scale from 0 to 2, where the mid-point of the patella in line with or lateral to the second toe was given a score of 0 ("good" movement quality), the mid-point of patella placed medially to the second toe was given a score of 1 ("fair" movement quality), and the mid-point of patella clearly placed medial to the first toe was given a score of 2 ("poor" movement quality). Any disagreement was resolved by consensus discussion between the two raters. If required, the recordings were reviewed 
Table 1. Characteristics of included participants

\begin{tabular}{|c|c|c|c|}
\hline & $\begin{array}{l}\text { ACLD } \\
(\mathrm{n}=13)\end{array}$ & $\begin{array}{l}\text { ACLR } \\
(\mathrm{n}=38)\end{array}$ & $\mathrm{p}$-value \\
\hline Age $\left(\right.$ years) ${ }^{\mathrm{a}}$ & $27 \pm 5.7$ & $23 \pm 5.2$ & $0.026^{*}$ \\
\hline $\operatorname{BMI}\left(\mathrm{kg} / \mathrm{m}^{2}\right)^{\mathrm{a}}$ & $24.8 \pm 3.0$ & $23.4 \pm 2.7$ & 0.112 \\
\hline Women n (\%) & $4 \pm 31$ & $19 \pm 50$ & 0.229 \\
\hline Injured knee right $\%$ & $8 \pm 62$ & $23 \pm 61$ & 0.949 \\
\hline Time since injury (weeks) ${ }^{b}$ & $21.29(16.6-52.6)$ & NA & NA \\
\hline Time since rec (weeks) ${ }^{\mathrm{b}}$ & NA & $27.8(19.7-47.1)$ & NA \\
\hline Associated injuries n \% & $9 \pm 69$ & $24 \pm 63$ & 0.662 \\
\hline Meniscal n \% & $5 \pm 38$ & $20 \pm 53$ & 0.333 \\
\hline Cartilage n \% & $2 \pm 15$ & $13 \pm 34$ & 0.198 \\
\hline Collateral ligament n \% & $4 \pm 31$ & $9 \pm 24$ & 0.663 \\
\hline Tegner activity level: Pre-injuryb & $9(7-9)$ & $9(8-9)$ & 0.253 \\
\hline Tegner activity level: Current ${ }^{\mathrm{b}}$ & $3(2.5-4)$ & $4(2.75-7)$ & 0.148 \\
\hline
\end{tabular}

*Statistically significant difference.

ACLD: anterior cruciate ligament deficiency; ACLR: anterior cruciate ligament reconstruction; ${ }^{a}$ : mean \pm standard deviation; ${ }^{\text {b: }}$ median (quartiles); rec: reconstruction; NA: not applicable.

Table 2. Quadratic weighted kappa for the knee position score reflecting the inter-rater reliability of the two assessors

\begin{tabular}{lc}
\hline Task & Quadratic weighted kappa \\
\hline Mini-squat & 0.46 \\
SLHD & 0.81 \\
COHD & 0.87 \\
\hline \multicolumn{2}{l}{ SLHD: single-leg hop for distance; COHD: cross- } \\
over hop for distance.
\end{tabular}

several times and/or in slow motion until consensus was reach. Visual assessments of medio-lateral knee position were valid against 2D and 3D kinematics ${ }^{30}$, and showed fair to excellent agreement (quadratic weighted kappa=0.46-0.87) (Table 2).

Data for individuals with ACLD and ACLR were analyzed separately. Spearman's rank correlation coefficient was used to evaluate any possible association between kinesthesia and the knee position scores during the tasks. The independent t-test and the Mann-Whitney U test were used to evaluate any possible differences in kinesthesia and the knee position score between individuals with ACLD and ACLR. Any correlations among participant demographics and kinesthesia and the knee position score were evaluated using Pearson's correlation coefficient or Spearman's rank correlation coefficient, as appropriate. For all correlations, Cohen's thresholds were applied: $\geq 0.1=$ small, $\geq 0.3=$ moderate, $\geq 0.5=$ large and $\geq 0.7=$ very large correlation ${ }^{31)}$. P-values $\leq 0.05$ were considered statistically significant.

\section{RESULTS}

Individuals with ACLD were approximately four years older compared to individuals with ACLR. No other demographic or kinematic differences were present. No significant associations were found between any of the demographic variables and the position of the knee or knee kinesthesia ( $p>0.05$ ). Sixteen participants ( 3 with ACLD and 13 with ACLR) were not able to perform the SLHD and 19 participants (4 with ACLD and 15 with ACLR) were not able to perform the COHD. Data for the knee kinesthesia and knee position score during the tasks for individuals with ACLD and ACLR are presented in Table 3.

Worse TDPM was significantly associated with a KMFP during the SLHD in individuals with ACLD $\left(\mathrm{r}_{\mathrm{s}}=0.662, \mathrm{p}=0.037\right)$. Worse TDPM was also moderately to highly correlated (but not statistically significant) to a KMFP during the mini squat and the COHD in this group, $\left(r_{s}=0.452, p=0.121\right.$ and $r_{s}=0.610, p=0.081$, respectively) (Table 4). No significant associations were observed between TDPM and the knee position score in individuals with ACLR $\left(\mathrm{r}_{\mathrm{s}} \leq 0.196, \mathrm{p}>0.05\right)$ (Table 4).

\section{DISCUSSION}

The main findings of this study indicated that worse knee kinesthesia may be associated with a KMFP during weightbearing tasks in patients with ACLD, whereas no such association was detected after ACLR. 
Table 3. Kinesthesia and the knee position score data for individuals with ACLD and ACLR

\begin{tabular}{|c|c|c|c|}
\hline & $\operatorname{ACLD}(n=13)$ & $\operatorname{ACLR}(\mathrm{n}=38)$ & ACLD vs. ACLR \\
\hline & \multicolumn{2}{|c|}{ Mean \pm SD } & p-value \\
\hline TDPM & $1.77 \pm 0.98$ & $2.28 \pm 1.18$ & 0.172 \\
\hline \multicolumn{4}{|c|}{ Median (quartiles) } \\
\hline Mini-squat & $0(0-0.5)(\mathrm{n}=13)$ & $0(0-0.25)(n=38)$ & 0.930 \\
\hline SLHD & $1(0-2)(n=10)$ & $1(0-1)(n=25)$ & 0.579 \\
\hline COHD & $1(0-2)(n=9)$ & $1(1-2)(n=22)$ & 0.841 \\
\hline
\end{tabular}

TDPM: threshold to detection of passive motion; ACLD: anterior cruciate ligament deficiency; ACLR: anterior cruciate ligament reconstruction; SLHD: single-leg hop for distance; COHD: cross-over hop for distance; SD: standard deviation.

Table 4. Correlation coefficients between kinesthesia and the knee position score during the functional tasks

\begin{tabular}{lccc}
\hline & Mini-squat & SLHD & COHD \\
\hline ACLD & $(\mathrm{n}=13)$ & $(\mathrm{n}=10)$ & $(\mathrm{n}=9)$ \\
TDPM & $\mathrm{r}_{\mathrm{s}}=0.452(\mathrm{p}=0.121)$ & $\mathrm{r}_{\mathrm{s}}=0.662(\mathrm{p}=0.037)^{*}$ & $\mathrm{r}_{\mathrm{s}}=0.610(\mathrm{p}=0.081)$ \\
ACLR & $(\mathrm{n}=38)$ & $(\mathrm{n}=25)$ & $(\mathrm{n}=23)$ \\
TDPM & $\mathrm{r}_{\mathrm{s}}=0.196(\mathrm{p}=0.238)$ & $\mathrm{r}_{\mathrm{s}}=0.030(\mathrm{p}=0.888)$ & $\mathrm{r}_{\mathrm{s}}=0.068(\mathrm{p}=0.757)$ \\
\hline
\end{tabular}

*Statistically significant correlation.

TDPM: threshold to detection of passive motion; ACLD: anterior cruciate ligament deficiency; ACLR: anterior cruciate ligament reconstruction; SLHD: single-leg hop for distance; COHD: cross-over hop for distance; $\mathrm{r}_{\mathrm{s}}$ : Spearman's rank correlation coefficient.

Worse kinetic acuity was significantly associated with a KMFP during SLHD in patients with ACLD. The correlations for the mini-squat and the COHD were also moderate to high $\left(\mathrm{r}_{\mathrm{s}}=0.452\right.$ and 0.610 , respectively $)$ in this group of patients, but the difference did not reach statistically significance. In correlation analysis, significance is highly dependent on sample size. That is, if the sample size is large as in epidemiological studies, even very small correlations will be significant, although the correlations may not be clinically relevant. On the other hand, if the sample size is small, the correlations have to be much higher to reach significance ${ }^{32}$. To reach significance for the correlations coefficients for the mini-squat and cross-over hop, 20 and 11 individuals, respectively were needed. The non-significant correlations for these tasks are, thus, most likely attributed to the small sample ${ }^{32)}$.

Afferent signals from mechanoreceptors included in the ACL were reported to play an important role for the gammamuscle spindle regulation and control of muscle stiffness during movements by providing the muscle spindles with proprioceptive information and thereby enhancing the stability of the joint during perturbation ${ }^{20}$. The results of this study suggest that adequate proprioception may be also important for controlling frontal plane knee motion during weight-bearing activity in patients with ACLD. This is in line with the results of our previous study, which revealed a relationship between worse kinesthesia and the KMFP during drop jump in participants with ACLD and ACLR ${ }^{22}$. In the current study, we did not find any association between kinesthesia and medio-lateral knee position in participants that had underwent ACLR. The reason for this discrepancy is not clear. ACL reconstruction was suggested to preserve the proprioceptive mechanoreceptors in the ruptured ACL and thereby retain the proprioception sense to some extent ${ }^{33)}$. Furthermore, a recent meta-analysis confirmed that patients with ACLD have worse proprioception than those with ACLR ${ }^{17}$. However, no differences in kinesthesia were found between the two groups in this study. One explanation for this discrepancy may be that all participants were high-level athletes before injury. Thus, both patients with ACLD and those with ACLR were well trained and most likely participated in the same amount of pre-injury prevention training including proprioceptive training. Therefore, further studies are needed to elucidate if a different treatment after ACL injury leads to different compensatory mechanisms that may explain this finding.

This study has several limitations. First, this was a hypothesis-generating exploratory study and the participants were not randomized to surgery or non-surgical treatment. Consequently, the result may be affected by selection bias in terms of different applied treatments (surgical/non-surgical). Second, the sample size in the ACLD group was quite small. This was mainly due to the fact that all participants were high-level athletes recruited at a sports injury clinic were ACLR is the most common treatment. Third, hop ability was not included in the inclusion criteria. This led to an even smaller sample size, since four participants in the ACLD group and 15 in the ACLR group were not able to perform both hop tests. Although the sample size was small, we found moderate correlations in patients with ACLD that may be of clinical importance. Forth, the small sample size also did not allow separate analyses of men and women in the two groups (ACLD/ACLR). A recent study reported that non-injured women have worse proprioceptive acuity compared to men ${ }^{34)}$. Furthermore, non-injured women and those with 
PFP were found to perform weight-bearing activities with increased 3D knee abduction compared to men ${ }^{35}$. Thus, the result might have been different if we had performed gender-stratified analyses. Despite these limitations, the results of this study indicate a role of proprioception, measured as kinesthesia, in movement quality of the knee in patients with ALCD. Finally, this cross-sectional study included only athletes with high pre-injury activity level. Hence, we can neither draw conclusions on any causal relationship, nor generalize obtained results to the general population.

The results of this study suggest a relation between worse kinesthesia of the knee and impaired ability to control frontal plane knee motion during weight-bearing activity, as assessed by visually observed KMFP in patients with ACLD. The lack of association between kinesthesia and medio-lateral knee motion in patients with ACLR indicate possible differences in sensorimotor function between patients with ACLD and ACLR that may be considered in training and rehabilitation regimens to prevent further injuries in these patients. Further studies, including randomized controlled trials, with greater sample size, are warranted to confirm these results.

\section{Funding}

This research did not receive any specific grant from funding agencies in the public, commercial, or not-for-profit sectors.

\section{Conflict of interest}

The author declares no competing interest.

\section{ACKNOWLEDGEMENT}

Author thanks Jenny Älmqvist Nae, MSc, and Gustav Nilsson, MSc, physical therapists at "Kulan Idrottsskadecentrum" in Malmö, for assessing and scoring the patients' movement quality.

\section{REFERENCES}

1) Yamazaki J, Muneta T, Ju YJ, et al.: Differences in kinematics of single leg squatting between anterior cruciate ligament-injured patients and healthy controls. Knee Surg Sports Traumatol Arthrosc, 2010, 18: 56-63. [Medline] [CrossRef]

2) Trulsson A, Garwicz M, Ageberg E: Postural orientation in subjects with anterior cruciate ligament injury: development and first evaluation of a new observational test battery. Knee Surg Sports Traumatol Arthrosc, 2010, 18: 814-823. [Medline] [CrossRef]

3) Goerger BM, Marshall SW, Beutler AI, et al.: Anterior cruciate ligament injury alters preinjury lower extremity biomechanics in the injured and uninjured leg: the JUMP-ACL study. Br J Sports Med, 2015, 49: 188-195. [Medline] [CrossRef]

4) Tengman E, Grip H, Stensdotter A, et al.: Anterior cruciate ligament injury about 20 years post-treatment: a kinematic analysis of one-leg hop. Scand J Med Sci Sports, 2015, 25: 818-827. [Medline] [CrossRef]

5) Hewett TE, Torg JS, Boden BP: Video analysis of trunk and knee motion during non-contact anterior cruciate ligament injury in female athletes: lateral trunk and knee abduction motion are combined components of the injury mechanism. Br J Sports Med, 2009, 43: 417-422. [Medline] [CrossRef]

6) Olsen OE, Myklebust G, Engebretsen L, et al.: Injury mechanisms for anterior cruciate ligament injuries in team handball: a systematic video analysis. Am J Sports Med, 2004, 32: 1002-1012. [Medline] [CrossRef]

7) Koga H, Nakamae A, Shima Y, et al.: Mechanisms for noncontact anterior cruciate ligament injuries: knee joint kinematics in 10 injury situations from female team handball and basketball. Am J Sports Med, 2010, 38: 2218-2225. [Medline] [CrossRef]

8) Kiapour AM, Demetropoulos CK, Kiapour A, et al.: Strain response of the anterior cruciate ligament to uniplanar and multiplanar loads during simulated landings: implications for injury mechanism. Am J Sports Med, 2016, 44: 2087-2096. [Medline] [CrossRef]

9) Kiapour AM, Kiapour A, Goel VK, et al.: Uni-directional coupling between tibiofemoral frontal and axial plane rotation supports valgus collapse mechanism of ACL injury. J Biomech, 2015, 48: 1745-1751. [Medline] [CrossRef]

10) Levine JW, Kiapour AM, Quatman CE, et al.: Clinically relevant injury patterns after an anterior cruciate ligament injury provide insight into injury mechanisms. Am J Sports Med, 2013, 41: 385-395. [Medline] [CrossRef]

11) Trulsson A, Roos EM, Ageberg E, et al.: Relationships between postural orientation and self reported function, hop performance and muscle power in subjects with anterior cruciate ligament injury. BMC Musculoskelet Disord, 2010, 11: 143. [Medline] [CrossRef]

12) Paterno MV, Schmitt LC, Ford KR, et al.: Biomechanical measures during landing and postural stability predict second anterior cruciate ligament injury after anterior cruciate ligament reconstruction and return to sport. Am J Sports Med, 2010, 38: 1968-1978. [Medline] [CrossRef]

13) Krosshaug T, Steffen K, Kristianslund E, et al.: The vertical drop jump is a poor screening test for ACL injuries in female elite soccer and handball players: a prospective cohort study of 710 athletes. Am J Sports Med, 2016, 44: 874-883. [Medline] [CrossRef]

14) Cronström A, Creaby MW, Nae J, et al.: Modifiable factors associated with knee abduction during weight-bearing activities: a systematic review and metaanalysis. Sports Med, 2016, 46: 1647-1662. [Medline] [CrossRef]

15) Hart JM, Pietrosimone B, Hertel J, et al.: Quadriceps activation following knee injuries: a systematic review. J Athl Train, 2010, 45: 87-97. [Medline] [CrossRef]

16) Klyne DM, Keays SL, Bullock-Saxton JE, et al.: The effect of anterior cruciate ligament rupture on the timing and amplitude of gastrocnemius muscle activation: a study of alterations in EMG measures and their relationship to knee joint stability. J Electromyogr Kinesiol, 2012, 22: 446-455. [Medline] [CrossRef]

17) Relph N, Herrington L, Tyson S: The effects of ACL injury on knee proprioception: a meta-analysis. Physiotherapy, 2014, 100: 187-195. [Medline] [CrossRef] 
18) Petersen W, Taheri P, Forkel P, et al.: Return to play following ACL reconstruction: a systematic review about strength deficits. Arch Orthop Trauma Surg, 2014, 134: 1417-1428. [Medline] [CrossRef]

19) Relph N, Herrington L: Knee joint position sense ability in elite athletes who have returned to international level play following ACL reconstruction: a crosssectional study. Knee, 2016, 23: 1029-1034. [Medline] [CrossRef]

20) Johansson H: Role of knee ligaments in proprioception and regulation of muscle stiffness. J Electromyogr Kinesiol, 1991, 1: 158-179. [Medline] [CrossRef]

21) Nagai T, Sell TC, House AJ, et al.: Knee proprioception and strength and landing kinematics during a single-leg stop-jump task. J Athl Train, 2013, 48: 31-38. [Medline] [CrossRef]

22) Cronström A, Ageberg E: Association between sensory function and medio-lateral knee position during functional tasks in patients with anterior cruciate ligament injury. BMC Musculoskelet Disord, 2014, 15: 430. [Medline] [CrossRef]

23) Roos PE, Button K, van Deursen RW: Motor control strategies during double leg squat following anterior cruciate ligament rupture and reconstruction: an observational study. J Neuroeng Rehabil, 2014, 11: 19. [Medline] [CrossRef]

24) Wikstrom EA, Song K, Pietrosimone BG, et al.: Visual utilization during postural control in anterior cruciate ligament-deficient and -reconstructed patients: systematic reviews and meta-analyses. Arch Phys Med Rehabil, 2017, 98: 2052-2065. [Medline] [CrossRef]

25) Cronström A, Roos EM, Ageberg E: Association between sensory function and hop performance and self-reported outcomes in patients with anterior cruciate ligament injury. Open Access J Sports Med, 2017, 8: 1-8. [Medline] [CrossRef]

26) Ageberg E, Flenhagen J, Ljung J: Test-retest reliability of knee kinesthesia in healthy adults. BMC Musculoskelet Disord, 2007, 8: 57. [Medline] [CrossRef]

27) Zätterström R, Fridén T, Lindstrand A, et al.: Rehabilitation following acute anterior cruciate ligament injuries-a 12-month follow-up of a randomized clinical trial. Scand J Med Sci Sports, 2000, 10: 156-163. [Medline] [CrossRef]

28) Noyes FR, Barber SD, Mangine RE: Abnormal lower limb symmetry determined by function hop tests after anterior cruciate ligament rupture. Am J Sports Med, 1991, 19: 513-518. [Medline] [CrossRef]

29) Myer GD, Schmitt LC, Brent JL, et al.: Utilization of modified NFL combine testing to identify functional deficits in athletes following ACL reconstruction. J Orthop Sports Phys Ther, 2011, 41: 377-387. [Medline] [CrossRef]

30) Nae J, Creaby MW, Cronström A, et al.: Measurement properties of visual rating of postural orientation errors of the lower extremity -A systematic review and meta-analysis. Phys Ther Sport, 2017, 27: 52-64. [Medline] [CrossRef]

31) Cohen J: Statistical power analysis for the behavioral sciences, 2nd ed. Hillsdales: Lawrence Erlbaum, 1988.

32) Bland M: An introduction to medical statistics. New York: Oxford University Press, 2000.

33) Papalia R, Franceschi F, Vasta S, et al.: Sparing the anterior cruciate ligament remnant: is it worth the hassle? Br Med Bull, 2012, 104: 91-111. [Medline] [CrossRef]

34) Muaidi QI: Does gender make a difference in knee rotation proprioception and range of motion in healthy subjects? J Back Musculoskeletal Rehabil, 2017, 30: 1237-1243. [Medline] [CrossRef]

35) Cronström A, Creaby MW, Nae J, et al.: Gender differences in knee abduction during weight-bearing activities: a systematic review and meta-analysis. Gait Posture, 2016, 49: 315-328. [Medline] [CrossRef] 